\title{
Les interruptions dans les débats médiatiques : une stratégie interactionnelle
}

\section{Hugues Constantin de Chanay et Catherine Kerbrat-Orecchioni}

\section{(2) OpenEdition}

\section{Journals}

\section{Édition électronique}

URL : http://journals.openedition.org/pratiques/1614

DOI : $10.4000 /$ pratiques. 1614

ISSN : 2425-2042

\section{Éditeur}

Centre de recherche sur les médiations (CREM)

\section{Édition imprimée}

Date de publication : 15 décembre 2010

Pagination : 83-104

\section{Référence électronique}

Hugues Constantin de Chanay et Catherine Kerbrat-Orecchioni, «Les interruptions dans les débats médiatiques : une stratégie interactionnelle », Pratiques [En ligne], 147-148 | 2010, mis en ligne le 15 décembre 2010, consulté le 19 avril 2019. URL : http://journals.openedition.org/pratiques/1614 ; DOI : 10.4000/pratiques. 1614 


\title{
Les interruptions dans les débats médiatiques : une stratégie interactionnelle
}

\author{
Hugues Constantin de Chanay, \\ Catherine Kerbrat-Orecchioni
}

ICAR, Université Lumière Lyon 2

\section{Problématique}

\subsection{L'interruption, une notion trop « ordinaire»?}

«Excusez-moi de vous interrompre », «Laissez-moi parler », « Il lui a coupé la parole sans arrêt $» . .$. : dans certains types d'interactions, et singulièrement dans les débats médiatiques qui nous intéressent en priorité, les interruptions sont un phénomène saillant, doté d'effets interactionnels puissants, auquel les participants (auditoire compris) sont particulièrement sensibles et qui donnent lieu de leur part à de fréquents commentaires. Or ce phénomène a été relativement peu traité dans la littérature relevant de l'analyse conversationnelle (dorénavant $\mathrm{CA}$ ), et cela depuis ses débuts, comme on le constate en parcourant les Lectures de Sacks (le mot d' " interruption » n'y fait que quelques apparitions éparses) ou le texte considéré comme fondateur de toute la réflexion sur le fonctionnement du système des tours, le célébrissime article de Sacks, Schegloff et Jefferson (SSJ 1974). On sait en effet que cet article s'emploie à dégager les quatorze principes régissant le turn-system, or le phénomène de l'interruption n'est mentionné qu'à propos du dernier de ces principes, et non pas en tant que tel mais au travers des divers procédés mis en œuvre pour « réparer » cette « violation » du système :

14. Repair mechanisms for dealing with turn-taking errors and violations obviously are available for use. [...] No special theoretical motivation is needed to observe that $[\ldots]$ the lore and practices of etiquette concerning " interruption » and complaints about it, the use of interruption markers such as " excuse me » and others, false starts, repeats or recycles of parts of a turn overlapped by others, as well as premature (i.e., before possible completion) stopping by parties to simul- 
taneous talk, are repair devices directed to troubles in the organization and distribution of turns to talk. (SSJ $1974: 39$ )

D’une manière générale, le mot d' « interruption » est rarement employé dans la littérature conversationnelle, et lorsqu'il l'est c'est le plus souvent avec une certaine réticence (que marque par exemple l'emploi des guillemets, comme dans la citation précédente), ce qui est d'autant plus remarquable que la littérature est au contraire fort prolixe sur la notion voisine d'overlap (même si l'on peut avoir des chevauchements de parole sans interruption et inversement, les deux phénomènes sont tout de même très généralement associés) ou sur les notions de transition relevant place (TRP) et de turn constructional unit $(\mathrm{TCU})^{(1)}$, qui sont elles aussi indissociables de celle d'interruption. Exception notable : l'article de Schegloff (2001) intitulé « Accounts of Conduct in Interaction : Interruption, Overlap, and Turn-Taking » où il est explicitement et largement question des interruptions, mais pour dénier à cette notion toute pertinence scientifique, ce qui nous permet d'y voir un peu plus clair dans les raisons de ce discrédit.

D'après Schegloff, le terme d' " interruption » (qui conserve ses guillemets tout au long de l'article) renvoie à une notion " ordinaire » ou « vernaculaire », qui n'a pas le même statut que les autres notions manipulées en CA : elle est en effet compromise avec l' " étiquette » et soumise à des considérations normatives, impliquant toujours un certain jugement de valeur - impossible de définir cet objet sans tenir compte de son caractère complainable : une interruption, c'est quelque chose dont on se plaint quand on la subit et dont on s'excuse quand on la commet ; c'est par ailleurs quelque chose qu'on ne peut traiter indépendamment du problème de l'appartenance catégorielle des participants (membership categorization, en particulier par rapport au facteur du « genre » et plus largement de la relation de pouvoir et de domination que les interruptions révèlent et construisent tout à la fois). Ce dont il ressort que la question de savoir si l'on a ou non affaire à une interruption est une question certes importante pour les participants à l'interaction, mais non pertinente d'un point de vue " académique " : « social scientific work based on this notion is problematic» (p. 289), car un tel travail est nécessairement entaché de considérations excessivement subjectives, voire moralistes.

À cette exclusive prononcée à l'encontre de la notion d'interruption on peut pourtant objecter qu'il peut sembler étrange, lorsque l'on revendique une sensibilité ethnométhodologique, de fonder cette exclusive sur le fait qu'il s'agit au départ d'une catégorie des «membres » (lesquels ne sont pas, pour reprendre le mot fameux de Garfinkel, des « idiots culturels ») ; qu'il revient au contraire aux linguistes de tenter de «techniciser» les intuitions communes sur la langue et le discours ; que l'identification d'une interruption a toujours quelque chose de « subjectif », à la différence il est vrai de celle des chevauchements dont l'existence est « objective », mais ni plus ni moins que celle des TRP et des TCU (voir par exemple Selting 2000 sur les raisons du caractère éminemment problémati-

(1) Pour désigner ces différentes notions nous avons fait le choix de retenir tantôt le sigle anglais (TRP, TCU), tantôt le sigle français (TP pour «tour de parole »), en fonction de l'usage qui nous semblait le plus fréquent en France. 
que et « unclear» de cette dernière notion). On ajoutera que l'on peut voir en filigrane dans le texte de Schegloff un autre motif de gêne vis-à-vis du phénomène de l'interruption : c'est qu'il met partiellement en déroute l'idée selon laquelle le talk-in-interaction se déroulerait de façon fondamentalement « ordonnée » et coopérative - on a vu que Sacks ne s'intéressait à l'interruption qu' au travers des moyens de sa réparation, et non comme constituant en elle-même une ressource au sein d'un ensemble de stratégies polémiques et de déstabilisation de l'adversaire ; Sacks qui insiste aussi sur le fait que les interruptions ne surgissent pas au hasard (" if you look at where interruptions occur in conversation, you'll find that they don't occur randomly », 1992 : 525), ce qui sera à vérifier dans notre corpus de débats, où l'on verra qu'il arrive aussi qu'elles surviennent de façon véritablement anarchique ( désordonnée ») dans des phases de dérèglement généralisé du système des tours.

En tout état de cause, même si elle pose des problèmes descriptifs délicats, cette notion est indispensable pour qui veut chercher à comprendre ce qui se passe dans les interactions, en particulier si l'on s'intéresse comme c'est notre cas aux interactions à caractère intrinsèquement confrontationnel telles que les débats médiatiques, où les interruptions jouent un rôle décisif dans la gestion du conflit et où le «style interruptif » des protagonistes constitue une composante non négligeable de leur « ethos ». La notion d'interruption, aussi floue et fluctuante soit-elle, est proprement incontournable pour l'analyse du discours en interaction. Notre objectif est donc ici de proposer quelques principes et outils pour tenter de rendre compte au moins partiellement d'un phénomène interactionnel éminemment pertinent.

\subsection{Travaux antérieurs sur la notion d'interruption}

Dans l'article précédemment cité, Schegloff se réfère aux travaux qui à la suite de la publication de l'étude de Zimmerman et West (1975) ont envisagé le phénomène des interruptions dans le cadre des gender studies, tendant à prouver que d'une manière générale, les hommes interrompent plus que les femmes, en relation avec leur position dominante dans la communication. Ce type d'approche a en effet donné lieu à une abondante littérature (voir, entre autres, Murray et Covelli 1988 ou Talbot 1992 pour une critique des travaux de West et Zimmerman, et Goldberg 1990 pour une revue de la littérature sur la question des interruptions comme marqueurs de dominance).

Par ailleurs, un certain nombre de travaux sur la communication politico-médiatique s'intéressent de façon centrale ou marginale au problème des interruptions, telles qu'elles se réalisent dans différents genres interactionnels, par exemple dans les interviews : Beattie 1982, comparant le «turn-taking style » de Margaret Thatcher, alors chef de l'opposition, et celui de Jim Callaghan, alors Premier ministre lors de deux interviews télévisées en avril 1979, montre ainsi que Thatcher interrompt relativement peu mais aussi cède difficilement quand elle est interrompue, avec des chevauchements qui vont jusqu'à 5 secondes, gagnant ainsi souvent la «battle for the floor». Mais la plupart des travaux dans ce domaine portent sur ces « guerres verbales » que sont les débats (surtout s'ils se déroulent en contexte électoral), les analystes décrivant alors les interruptions comme des composantes de la dimension polémique de l'interaction : Hutchby 
1992 (à partir d'un corpus de débats radiophoniques londoniens) insiste sur le caractère confrontationnel des interruptions dans ce contexte ; Luginbühl 2007 (à partir de l'étude d'une émission suisse de débats politiques télévisés) envisage l'interruption comme un élément de la violence conversationnelle ; Agha 1997 (à partir de l'analyse du débat présidentiel entre Bill Clinton et Bob Dole en octobre 1996) analyse les out-of-turn utterances comme des procédés au service de la rhétorique de l'agression. Pour le domaine français, signalons deux études s'employant elles aussi à comparer le comportement interruptif des deux protagonistes d'un débat électoral : celle de Trognon et Larrue (1994 : 63-66) sur les duels Chirac-Fabius (1985) et Chirac-Mitterrand (1988); et celle plus récente et précise à la fois de Sandré (2009) sur le débat entre Ségolène Royal et Nicolas Sarkozy lors de l'entre-deux-tours des présidentielles (2 mai 2007) : tout en reconnaissant les difficultés qu'il y a à identifier un phénomène qui n'est « pas un donné mais une reconstruction de l'analyste» (p. 70), l'auteure nous fournit des indications précises sur le nombre des interruptions effectuées de part et d'autre (148 pour SR, soit $35 \%$ de ses prises de parole vs 132 pour NS, soit $30 \%$ seulement) puis sur la proportion des interruptions à visée polémique (la très grande majorité) qu'elle oppose aux interruptions à fonction de gestion de l'interaction (c'est le cas surtout de celles effectuées par les animateurs) et enfin aux interruptions coopératives (qui seraient moins de 3\%). Quoique portant sur ce même débat (dorénavant «Sarkolène »), notre approche sera un peu différente de celle de Sandré car il nous semble que ce type de relevé, assurément utile, dépend à un tel point de choix analytiques qu'il est indispensable de problématiser la notion d'interruption avant de procéder à l'analyse des données, ce que nous tenterons de faire avec l'aide des outils élaborés dans le cadre de l'analyse conversationnelle. Nous tenterons par là même de combler le fossé qui sépare d'un côté, les tenants de la CA qui pour la plupart évitent de recourir à la notion d'interruption, et de l'autre les analystes du discours des médias qui y recourent abondamment mais sans toujours suffisamment insister sur les problèmes qu'elle soulève.

Mentionnons pour terminer quelques contributions susceptibles de nous aider dans cette entreprise :

- Lerner (1989) s'intéresse au fait qu'à côté des transferts « smooth » de la parole d'un current speaker (A) à un next speaker (B), il arrive que le candidat à la succession démarre out of turn; l'article envisage alors ce qui se passe après ces intervening utterances, différents cas de figure pouvant être distingués : ou bien A perd le tour ou il le récupère ; dans ce dernier cas deux possibilités se présentent, l'unperturbed continuation ${ }^{(2)}$ (A poursuit son énoncé comme si de rien n'était) et la delayed completion (c'est-à-dire l'achèvement par A de son tour provisoirement interrompu par B, l'énoncé de A étant alors discontinu, et pouvant ou non intégrer certains éléments du contenu de l'énoncé interruptif). Ces différents cas de figure seront examinés à la lumière de notre corpus. Pour l'instant citons cette « remarque conclusive » de l'article de Lerner (1989: 175) :

Delayed completion is [...] one procedure for resolving overlap begun by interja-

(2) Ce fonctionnement s'apparente à ce que Trognon (1988:72-73) nomme la « connexion saltatoire », dans laquelle tout de passe comme si « le premier locuteur agençait un monologue en oubliant ce qu'a dit son interlocuteur ». 
cent entry into a turn by a next speaker. [...] it is a device [...] apparently used to minimize overlapping talk by requiring a speaker to drop out the onset of another speaker's utterance.

Cette affirmation est en effet révélatrice de la représentation dominante que l'on se fait en CA du talk-in-interaction : tout y serait mis au service du bon fonctionnement de la machine conversationnelle, ramené au bon fonctionnement du système des tours. Mais pour minimiser le chevauchement, la solution la plus simple n'est-elle pas que A renonce complètement à son tour? S'il ne le fait pas, préférant poursuivre son tour après ce petit délai imposé par l'interruption, c'est que la delayed completion, plus encore qu'un procédé de résolution du chevauchement, est un moyen de récupérer le «floor». D'ailleurs, si le seul but des sujets conversants était que «ça marche », il n'y aurait pas d'interruption (si ce n'est par inadvertance, l'interrupteur se retirant aussitôt qu'il a compris sa bévue). Le phénomène des interruptions a précisément pour intérêt de montrer que dans la conversation l'enjeu n'est pas seulement de faire tourner au mieux la machinerie des tours, mais qu'il existe au-delà de ces considérations techniques toutes sortes d'enjeux d'un autre ordre (en particulier relationnel), ce que du reste Lerner admet puisqu'il suggère un peu plus loin : " It might be useful to include the activity of interactionally dubbing another as an interrupter in the examination of the conversational activies that constitute relationships. "

- Guillot (2005), dans un article qui aborde de front la notion d'interruption, montre combien cette notion est à la fois problématique (car certaines divergences interprétatives peuvent intervenir à tous les niveaux) et nécessaire, en particulier pour les recherches contrastives (car la gestion des interruptions varie d'une culture à l'autre, ce qui peut entraîner certaines difficultés dans la communication interculturelle).

- L'article de Schmale (2008) est surtout centré sur la notion d' "UCT » (forme francisée du sigle anglais «TCU»), mettant en évidence le fait que ce qui est pertinent pour une telle unité c'est qu'elle soit interactivement achevée, ce qui n'implique pas forcément qu'elle le soit syntaxiquement. La réflexion est menée à partir de l'étude d'un corpus de conversations téléphoniques où sont détectés différents cas de figure de ce phénomène d'achèvement interactif sans achèvement syntaxique. Elle aboutit à cette conclusion (p. 830) que le véritable problème n'est pas de déterminer rétrospectivement les UCT, mais de « reconstituer à travers une analyse séquentielle des activités réciproques des participants, les endroits que ces derniers traitent comme pertinents à la transition (PPT) en prenant effectivement la parole. " Nous ne pouvons qu'être d'accord, sauf à ajouter que le fait que $B$ prenne la parole en un point $X$ n'est pas forcément une preuve que $\mathrm{X}$ est pour $\mathrm{A}$, et même pour $\mathrm{B}$, «pertinent à la transition », du fait justement de la possibilité de cette violation du système que constitue l'interruption... Possibilité d'ailleurs discrètement évoquée plus loin, lorsque l'auteur précise que les UCT peuvent englober toutes sortes de constructions, syntaxiquement achevées ou non, qui donnent lieu à une prise de parole « sans que cette dernière ne constitue une interruption du partenaire »-mais tout le problème est précisément là ! On voit combien les interruptions viennent perturber non seulement le déroulement de l'échange mais aussi la modélisation du turn-system, puisqu'il ne suffit pas de regarder à quels moments et dans quelles conditions se 
font les changements de locuteurs pour pouvoir reconstituer les règles qui les permettent étant donné que ces règles sont loin d'être systématiquement respectées. On voit aussi combien sont indissociables les notions de TCU/UCT et d'interruption, puisqu'un TCU est un segment de tour susceptible de donner lieu en son (quasi-)terme à un changement de locuteur sans que ce changement produise l'effet d'une interruption.

\subsection{L'interruption : définition et problèmes}

\subsubsection{Définition}

En contexte interactionnel, le flux discursif s'organise de telle sorte que s'y trouvent inscrits par le locuteur en place (A) certains Possible Completion Points marquant une Transition Relevance Place. L'auditeur va donc guetter et anticiper l'apparition de ces TRP afin de pouvoir prendre la parole sans laisser de blanc (minimization of gap), mais il ne doit pas non plus prendre la parole trop tôt afin de ne pas occasionner un chevauchement de parole prolongé (minimization of overlap) : voilà pour le principe. Mais dans les faits, il arrive fréquemment que B s'empare de la parole alors que A n'a apparemment aucune intention de la lui léguer: on dit alors que B « interrompt» A (il lui «coupe » la parole),

- soit parce que B prend pour un TRP effectif ou imminent ce qui ne l'est pas pour A : l'interruption est involontaire ;

- soit parce que B intervient délibérément en dehors de tout TRP possible.

Pour les participants, les interruptions mettent donc en jeu les attentes et intentions de A ainsi que les hypothèses que fait B sur les intentions de A ; corrélativement, il s'agit pour l'analyste de tenter de reconstituer ces intentions et hypothèses à partir d'indices concernant la présence projetable d'un TRP.

Une interruption peut donc être définie comme une prise de parole par un nouveau locuteur en dehors de tout TRP. Or les deux ingrédients de la définition censée servir de base à l'identification du phénomène peuvent dans bien des cas poser problème.

\subsubsection{Problèmes d'identification des interruptions}

(1) Y a-t-il ou non « prise de parole »?

Il est généralement admis que ne constituent pas de véritables prises de parole les productions à fonction de back-channel (« régulateurs » ou « continueurs»). Mais le problème se pose plus largement pour les collaborative utterances, les interventions à fonction de "coénonciation 》 ou les activités de " soufflage » - on peut en effet estimer qu'un soufflage ne constitue une interruption qu'à partir du moment où il n'est manifestement pas sollicité par le locuteur en place, le regard jouant dans cette affaire un rôle décisif(Goodwin et Goodwin 1986). Il se pose aussi en cas de side séquence (Jefferson 1972), comme dans cet exemple mentionné par Murray et Covelli (1988:109) :

\footnotetext{
Husband: I think [ that

$\rightarrow$ Wife : [Do you want some more salad?

Husband: No I wan- (trailing off) -ted to say [...]
} 
Ayant soumis à des tests de réception un certain nombre d'échantillons de conversations, Murray et Covelli ont ainsi constaté que les phénomènes de ce type étaient responsables de la plupart des divergences concernant les jugements d'interruption portés par leurs informateurs.

(2) Cette prise de parole se fait-elle « en dehors de tout TRP»?

La projection d'une fin de tour convoque des indices de nature non seulement syntaxique (indices excessivement privilégiées par la CA en ses débuts) mais aussi et peut-être surtout prosodiques (éventuellement mimogestuels) et sémantico-pragmatiques (nature de l'acte de langage réalisé par l'énoncé, orientation argumentative - une structure « concessive » laissant par exemple présager un «mais » et sa suite...) : les TRP sont en fait des CTRP (complex transition-relevance places, Ford et al. 1996) reposant sur un faisceau d'indices diversement hiérarchisés. Ajoutons qu'il est également nécessaire de prendre en compte le type d'interaction et d'activité en cours, comme on le voit dans le cas limite de la conférence magistrale, où les règles du genre interdisent l'intervention de tout " successeur» : pas question donc de chercher à identifier le moindre TRP dans un discours oral de ce type, qui se décompose bien en unités syntaxiques et macro-syntaxiques, mais point en TCU, ces unités spécifiquement interactionnelles n'existant que dans la perspective d'une possible alternance des tours.

Il n'est donc pas toujours aisé d'identifier un TRP effectif, et l'on verra que la notion ne peut être traitée qu'en termes graduels : un TRP est plus ou moins évident, une interruption peut donc être perçue comme plus ou moins "sévère " (Murray et Covelli 1988). Mais en outre, ces points de transition sont anticipables, c'est-à-dire qu'un enchaînement « prématuré » peut être « conditionnellement pertinent »- mais dans quelles conditions ?

Talk which overlaps a current speaker's talk may be recognized as « interruptive » if initiated nowhere near a possible completion, and as enthusiastic if overlapping what has already be recognized as its incipient possible completion. (Schegloff $1992: 117-118$ )

Passons sur la distinction entre intervention « interruptive » et « enthousiaste »; mais que faut-il entendre par «near a possible completion »? À cette question de nombreuses études se sont depuis lors employées à apporter des éléments de réponse, notamment en identifiant certains types de segments susceptibles d'être chevauchés par anticipation d'un TRP - tels ces « lexèmes stéréotypés », « formules d'adresse », «phrasillons » et autres « séquences très brèves en fin de tour sans conséquence au niveau des activités conversationnelles » qu'étudie Schmale 2002 -, corroborant l'idée que les locuteurs analysent finement le tour :

Si le fait que le locuteur incipient démarre son tour exactement à la fin du précédent constitue une première évidence de son analyse de la structuration du tour [...], le fait qu'il puisse le faire en anticipant la fin du tour précédent en est une encore plus forte. (Mondada $2007: 15$ )

- mais la remarque ne vaut qu'à proximité d'un TRP : sinon l'anticipation est plutôt la preuve, soit de son incapacité à projeter correctement la fin du tour, soit d'une parfaite indifférence aux TRP... 
Ce qui est en tout cas certain, c'est d'une part que ce problème ne peut être traité en termes purement mécaniques, à la manière de Zimmerman et West définissant d'abord l'interruption comme une prise de parole survenant «plus d'un mot » avant un point de complétion possible, puis remplaçant quelques années plus tard le critère du mot par celui des « deux syllabes », ce qui ne vaut guère mieux : il est indispensable de s'appuyer sur des critères qualitatifs, concernant entre autres le degré de prévisibilité ou d'informativité de la fin d'énoncé ; et d'autre part que les notions de TCU, de TRP et d'interruption sont absolument indissociables : 1'existence des interruptions est en quelque sorte la preuve $a$ contrario de l'existence des « points de transition pertinents », notion qui n'a d'intérêt que dans la mesure où elle vise à expliquer pourquoi certaines prises de tour sont en quelque sorte légales et «conditionnellement pertinentes », quand d'autres - les interruptions - ne le sont pas (ou le sont moins).

\subsubsection{Notre approche}

Nous défendrons ici l'idée qu'il est possible d'étudier les interruptions avec les instruments de la CA, en reprenant les notions de tour de parole (TP), de turn constructional unit (TCU), de transition relevance place (TRP), et en proposant d'ajouter une nouvelle unité, le segment de tour interrompu (STI). Nous avons déjà eu l'occasion de présenter et d'utiliser brièvement cette unité pour évaluer la plus ou moins grande résistance des locuteurs à l'interruption, par comparaison du nombre de tours et du nombre de STI (Constantin de Chanay et KerbratOrecchioni 2007 : 318). Le présent article nous fournira l'occasion d'y revenir d'une manière plus fouillée et plus technique.

Nous avons retenu pour cela trois corpus :

- le débat entre Ségolène Royal (SR) et François Bayrou (FB) diffusé sur BFM TV le 29 avril 2007 : la manière très courtoise dont un journaliste (Olivier Mazerolle, OM) s'efforce de reprendre la parole à une intervenante très volubile permettra d'étudier le placement optimal des interruptions (optimal du point de vue du respect des règles de l'alternance des tours, même si les interruptions ont toujours, par définition, un certain caractère transgressif par rapport à ces règles);

- un numéro de l'émission Le temps de choisir diffusé sur LCP le 4 avril 2007 avec en invité Philippe de Villiers (PhdV), qui comporte un épisode de crise durant lequel l'invité sort de ses gonds, et qui permettra d'étudier des placements d'interruptions qui ne s'appuient au contraire apparemment sur aucune analyse du tour de l'autre ;

- enfin, notre corpus principal : le débat «Sarkolène » diffusé le 2 mai 2007 sur France 2 et TF1, opposant les deux candidats à l'élection présidentielle Ségolène Royal (SR) et Nicolas Sarkozy (NS), qui combine des caractéristiques des deux précédents.

Nous nous pencherons dans un premier temps sur les problèmes de segmentation en empruntant des exemples aux trois corpus, avant de proposer une étude rapide des interruptions dans le troisième. 


\section{Les interruptions : approche sur corpus}

\subsection{Interruptions et problèmes empiriques de segmentation}

\subsubsection{Suivi des tours et STI}

L'existence de segments de tours interrompus (STI) s'impose logiquement dès lors que l'on admet que des tours peuvent se poursuivre au-delà de zones de chevauchements interruptifs. S'il nous semble pertinent de les identifier, c'est parce qu'un tour qui se poursuit malgré une intervention adverse n'a plus exactement le même statut après l'interruption qu'avant : c'est un tour qui se maintient pour ainsi dire de force malgré l'intervention « indue ». Les segments situés de part et d'autre du chevauchement sont alors des STI.

On peut se demander si la zone de chevauchement dans le tour constitue un segment à part, ou si elle appartient à l'un ou l'autre des deux STI (antérieur et postérieur), et si oui, auquel. Cette zone de chevauchement étant rarement homogène, cela n'a pas grand sens d'en faire un segment à part. Dans l'exemple suivant (Sarkolène 01.56.30) :

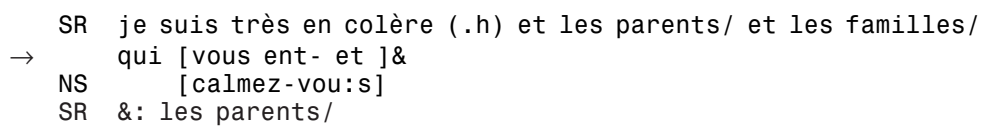

le segment chevauché comporte un premier volet « vous ent- » qui semble poursuivre ce qui précède, et un second « et » qui initie la reprise. Ces réactions sont bien sûr à décrire dans leur déroulement temporel précis. Mais ce qui nous intéresse ici dans un premier temps, c'est que le tour se poursuive ou non après le chevauchement : c'est cela qui semble caractéristique des locuteurs les plus « coriaces »-alors que les chevauchements et perturbations se rencontrent aussi dans le cas où les locuteurs cèdent facilement la parole. Aussi le plus logique est-il de fusionner le segment chevauché avec le segment précédent pour en faire un seul et même STI. Ce choix respecte l'intuition selon laquelle lorsqu'il y a une interruption, elle partage la chose interrompue en deux.

On peut dire dès lors que, lorsque A est interrompu par $\mathrm{B}^{(3)}$ :

- si A cède son tour, on a $n$ STI pour $n$ interruptions, avec $n>1$ lorsque B a dû intervenir plusieurs fois pour parvenir à ses fins ; cas particulier : lorsque $n=1,1 \mathrm{STI}=1$ tour (tour « mono-segment »);

- si A résiste et conserve son tour, on aura $n+1$ STI pour $n$ interruptions.

Cela étant, les cas concernés les plus fréquents sont typiquement de forme :

(3) Nous tenons pour négligeables les cas (rarissimes) d'interruptions sans chevauchement, qu'il serait d'ailleurs peu judicieux de monter en épingle - ce sont des artefacts créés par le fait de ne considérer que le verbal, alors que dès que l'on prend en compte le mimo-gestuel, il y a bel et bien chevauchement (voir p. ex. Mondada 2004 et 2007 pour une extension de la notion de tour au-delà de la zone strictement définie par la seule activité de parole). 
c'est-à-dire qu'on a majoritairement des STI quand A résiste à l'interruption de $\mathrm{B}$ : chose extrêmement courante dans les débats politiques, où les interruptions ne réussissent pas du premier coup et où il faut faire le «forcing » pour s'imposer. Le nombre des STI par rapport aux TP donne des indications sur la capacité des interactants à insister aussi bien qu'à résister.

La solution que nous avons retenue dans la transcription pour les dénombrer consiste à numéroter les TP et à repérer en début de paragraphe les STI par une lettre de l'alphabet associée à ce numéro de tour. En pratique, cela n'est pas redondant avec l'usage de l'esperluette en début et en fin de ligne : le changement de paragraphe « intra-tour » peut se produire de manière contingente au sein d'un même STI lorsque le transcripteur arrive en fin de ligne; dans ce cas on conservera en début de ligne la même " désignation de tour » (numéro ou numéro + lettre), augmentée du signe «'».

\subsubsection{Interruptions et organisation en TCU}

Problème : si les interruptions « don't occur randomly » (Sacks 1992 : 525), à la limite ce ne sont plus des interruptions, mais une forme d'intervention interactivement adaptée. Analysant un extrait de débat médiatique, Mondada (2001: 148) soutient ainsi qu'il n'y a pas de perturbation du système des tours, la place des interventions attestant au contraire l'analyse du tour en cours par les participants, l'un prenant la parole «systématiquement aux frontières des unités qui construisent [le] tour [de son interlocuteur] », l'autre cumulant des unités syntaxiques de manière à « minimiser la pertinence des points possibles de transition ».

Mais en va-t-il de même dans tous les cas? Non. Nos deux premiers corpus présentent de ce point de vue deux cas extrêmes.

Le débat Bayrou-Royal contient nombre d'interventions interruptives qui tiennent compte dans une certaine mesure de l'organisation en TCU du tour interrompu, en particulier de la part du journaliste OM. Du fait de son rôle, celui-ci doit se montrer très respectueux : il lui est donc difficile d'intervenir si SR ne produit pas de TRP. SR quant à elle s'arrange au contraire pour différer au maximum ces TRP, ou les minimiser. Examinons-en rapidement un extrait (dans lequel nous avons pratiqué quelques coupures indiquées par $[\ldots])^{(4)}$ :

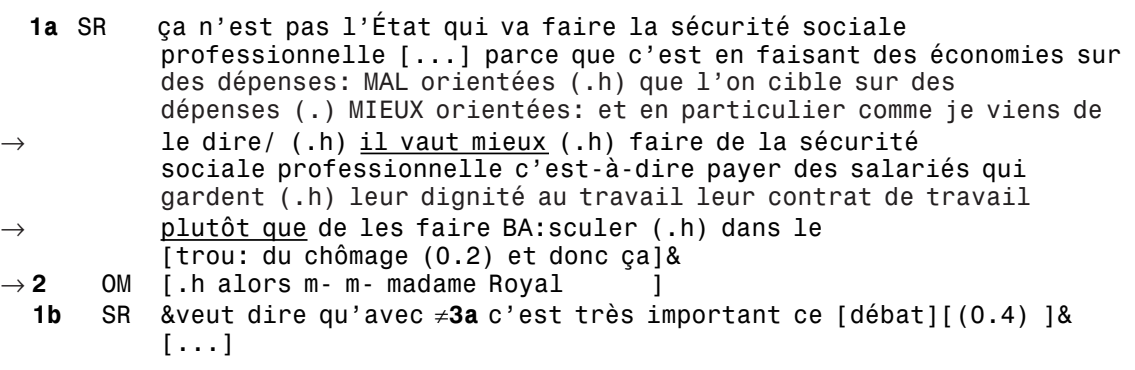

(4) Nos transcriptions, et la segmentation en STI qu'elles proposent (1a, 1b...), résultent de nombreuses décisions analytiques prises sur la base de considérations syntaxiques (en tenant compte de la prosodie et des gestes) et pragmatiques, ce qu'il est impossible de détailler ici. 


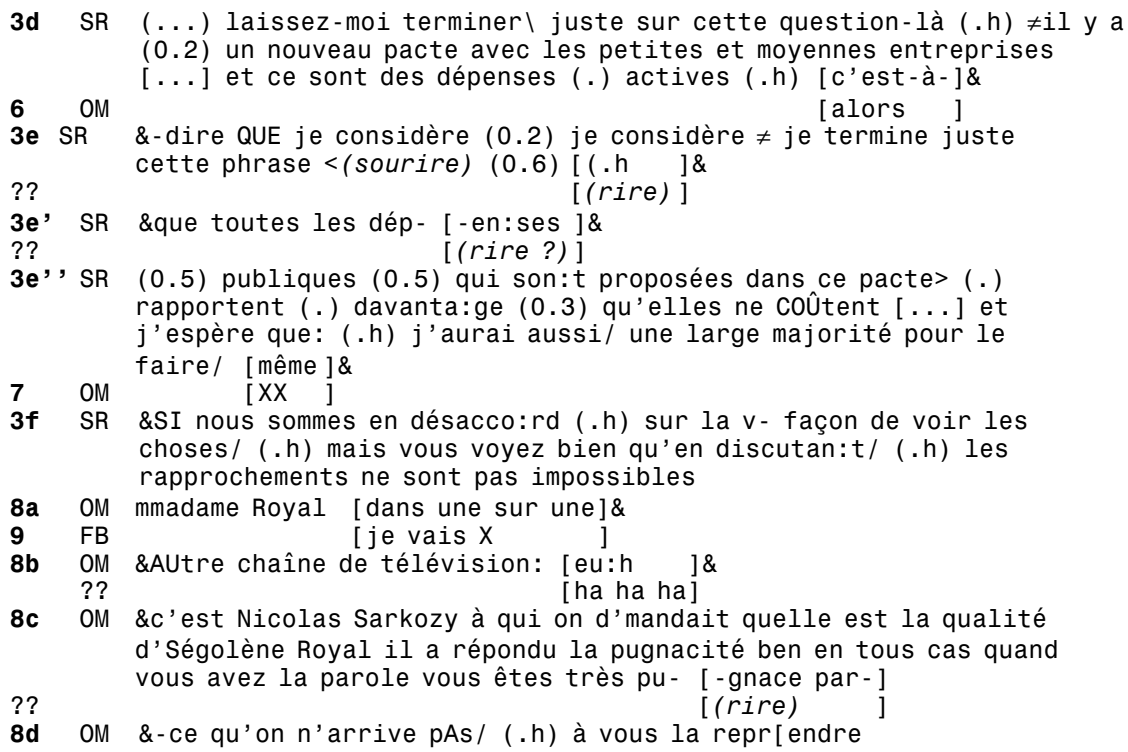

Bien qu'elle prenne en compte l'intervention d'OM en 3a, SR ne rend véritablement les armes qu'à la fin de $3 \mathrm{f}$ (soit deux bonnes minutes après la première tentative d'OM) et elle occupe le terrain par de longs passages monologaux (1a, $3 \mathrm{~d}, 3 \mathrm{e}$ "), produisant de longs TCU qui diffèrent syntaxiquement des TRP (ex. en 1a « il vaut mieux... plutôt que... »), ou les minimisant, par exemple en renforçant l'intensité ( 3 e et $3 \mathrm{f}$ ), ou en enchaînant immédiatement comme en 1a en fin de TCU, par un procédé qui s'apparente à celui du rush trough (Schegloff 1987 : 77) :

(...) MIEUX orientées: et en particulier comme je viens de le dire/ (.h) il vaut mieux $(\ldots)$

ce en quoi l'on peut voir un exemple de divergence entre les indices syntaxiques et les indices prosodiques de TRP, SR tâchant par ces derniers de rectifier online le caractère potentiellement transitionnel des frontières projetées par les premiers.

OM est quant à lui très tenace, mais ses interruptions n'arrivent cependant pas « au hasard» (du moins au tout début, car il est facile de voir que plus l'interaction progresse, moins il profite d'éventuels TRP) : à preuve, il se tient coi pendant les passages monologaux de $1 \mathrm{a}, 3 \mathrm{~d}, 3 \mathrm{e}$ ", ; il avoue ses difficultés (8d), lesquelles ne sont autres que des difficultés à intervenir alors que n'est produit aucun TRP. Il suffirait sans cela de continuer à parler comme un sourd et de jouer à qui va s'arrêter le dernier, comme on va le voir immédiatement dans le second corpus.

Dans les débats de ce type, les participants peuvent en effet prendre la parole n'importe quand ou presque. Soit ce moment de « crise» au cours de l'émission Le temps de choisir, alors que la journaliste Emilie Aubry (EA) vient de provoquer chez PhdV une fureur durable en citant in extenso son patronyme pour rappeler ses « lettres de noblesse». S'ensuivent deux bonnes minutes d'altercation, avec de très longs chevauchements qui sont de véritables défis à la transcription : 
impossible de parvenir à une version lisible tout en linéarisant. Nous avons donc finalement opté pour une présentation en colonnes :

PhdV

EA

\begin{tabular}{|c|c|}
\hline $\begin{array}{l}\text { euh vous feriez le: le musulman untel/ } \\
\text { le juif untel/ et caetera/ s vous trou- } \\
\text { vez/ ( } 0.2) \text { i faut que j'porte une } \\
\text { étoile jaune/ ( } 0.7) \text { qu'est-ce que ça } \\
\text { veut dire ça vicomte }\end{array}$ & \\
\hline $\begin{array}{l}\rightarrow \quad \text { [attendez (0.4) XXX (vous vous) } \\
\text { où est-ce que vous avez vu: où est-ce } \\
\text { que vous avez vu: attendez attendez ma- } \\
\text { dame (0.2) (mais) regardez-moi dans les } \\
\text { yeux où est-ce que vous avez pris ça: } \\
(0.2) \text { où est-ce que }(0.3) \text { attendez où } \\
\text { est-ce que vous avez pris ça: (0.3) et } \\
\text { et où est-ce que vous avez tiré ça (.) } \\
\text { (d'ailleurs) et c'est c'est quoi c'est } \\
\text { une émission règlement d'com:ptes } \\
\text { c'est pas un c'est pas une é-] }\end{array}$ & $\begin{array}{l}\text { [vous faites des amalgames } \\
\text { étran:ges j'aurais rappelé le vérita- } \\
\text { ble nom de n'importe quel inv- PAS } \\
\text { d'polémique sur ce sujet (0.2 j'vous } \\
\text { prêterai les portraits (.) j'vous prê- } \\
\text { terai les portrai:ts }(0.2) \text { j'vous prê- } \\
\text { terai les portrai: :ts dans L'Express } \\
\text { notamment (h. 0.3) ce sont des choses } \\
\text { qui reviennent souvent parce que ça } \\
\text { fait partie d'votre personnage }(0.2) \text { je } \\
\text { vous passerai l'dossier d'presse] }\end{array}$ \\
\hline [-mission politique] & $\neq[$ absolument pas $]$ \\
\hline
\end{tabular}

Immédiatement après « vicomte » démarre un chevauchement qui se poursuit sur 17 secondes - et il y en aura d'autres ! On a affaire à un cas limite : la superposition. Peut-on encore parler d'interruptions? C'est douteux : on peut objectivement délimiter des «segments de flux de parole » grâce aux pauses « intra-STI », mais on ne peut pas vraiment les regrouper en STI car aucun de ces segments n'est vraiment « de part et d'autre » d'une interruption. On aurait plutôt deux grands STI, et c'est bien l'impression qu' on a au fond : celle que PhdV et EA parlent de manière « ininterrompue », se fichant éperdument de la présence ou de l'absence de TRP (ce qui est plus étonnant encore de la part de la journaliste). Les participants semblent ne «suivre le tour» de l'autre que pour s'assurer qu'ils ne laissent pas passer quelque chose de trop important - ils gardent une oreille. C'est ce qui explique le retour à l'interaction avec le « absolument pas » final, en réaction immédiate à «c'est une émission règlement de comptes ». L'adaptation interactive des interruptions est donc très variable selon les corpus, et plus précisément selon les phases considérées au sein de ces corpus : les moments de crise sont à part, et toute l'émission Le temps de choisir n'est pas du même tonneau (le retour au calme coïncide avec un retour au respect relatif des règles de l'interaction). Tout porte à croire que si les participants cherchent à ne pas intervenir «au hasard », c'est essentiellement pour des raisons de politesse, qui les poussent à faire des interruptions « discrètes » et n'ayant pas trop l'air d'en être : ce qui est assurément graduel.

Comment dès lors rendre compte de cette gradualité qui caractérise le sentiment d'interruption? Il semble qu'elle tienne à un certain nombre de facteurs, parmi lesquels la présence ou non d' « adoucisseurs » de l'intervention interruptrice, son but (a-t-elle ou non vocation à ravir le tour?), la plus ou moins grande netteté des indices de TRP (continuum entre l'imminence avérée et l'absence patente), la plus ou moins grande imminence de ces TRP, etc., ainsi que la possibilité pour certains indices de suggérer des places transitionnelles qui ne sont cependant pas des TRP « à part entière » (Sarkolène 00.43.02) : 


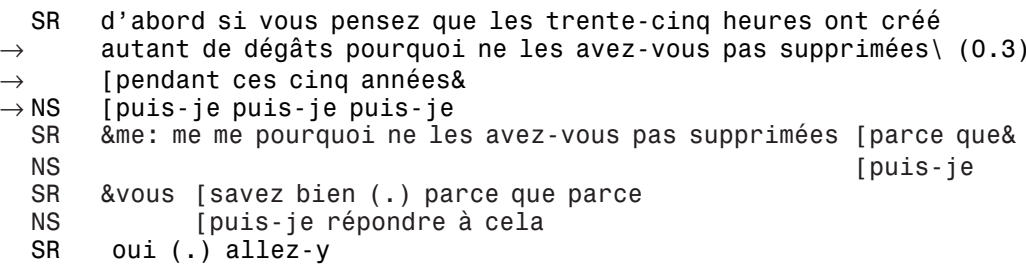

On pourrait penser que sur la base de l'apparente complétude syntaxique, ainsi que de l'intonation descendante et de la pause, le silence de SR après une question rhétorique est interprété comme un TRP par NS, qui tente de prendre le tour ; mais il le fait par une formulation qui présuppose que pour lui, il n'y a pas de TRP (pourquoi sinon demander la permission?), et de fait les expansions produites ensuite par SR semblent confirmer que pour elle non plus, il n'y en avait pas.

Mais on pourrait en outre distinguer des « niveaux » de construction du tour : les participants ne doivent pas seulement construire des TCU, mais aussi accomplir des tâches plus globales s'étendant sur plusieurs TCU, comme traiter une question ou mener à bien une argumentation. On pourrait ainsi distinguer entre deux niveaux au moins, l'un « local», celui des TCU, et un autre plus « global», auquel se déterminent ce que certains appellent des tours « multi-units » (Ford \& Thompson 1996, Selting 2000). Schmale (2008: 823 et 826) fait lui aussi une distinction de cet ordre lorsqu'il envisage la possibilité d'énoncés pragmatiquement incomplets à un niveau local mais complets à un niveau global. On pourrait ainsi distinguer des TRP locaux (fin de TCU) vs globaux (fin de tâche); les seconds ont une place beaucoup plus libre, ils sont plus «durables »- et c'est pourquoi ils peuvent précéder et même rendre inutile l'achèvement de TCU " locaux » (cas examiné par Schmale).

La légitimité de l'interruption n'est pas la même selon le niveau, global ou local, de ce qu'elle contrarie ou de ce qu'elle épaule. À gros traits, on peut avoir quatre cas de figure :

- contrariété locale + globale : interruption maximale, ex. PhdV qui « bloque » le déroulement de l'émission;

- contrariété locale mais coopération (ou non contrariété) globale : information ou contestation ponctuelles; ou encore, cas de NS attendant patiemment que SR ait fini son réquisitoire (scène de la "saine colère " dans Sarkolène) pour lancer une interruption, qui passe alors pour plus légitime (son «calmez-vous » a dû arriver à point nommé pour le spectateur qui ne l'a pas considéré comme interruptif voir infra);

- coopération locale mais contrariété globale : tentative polie pour reprendre le tour (ex. OM dans le corpus Bayrou-Royal) ; mais aussi enchaînements indésirables ou pseudo-enchaînements - ex. interruptions commençant par «alors » (OM toujours dans le corpus Bayrou-Royal), qui font « comme si » il y avait un TRP. ;

- coopération locale + globale : régulateurs, interventions « supportives » (qui, comme on va le voir, sont rares dans Sarkolène. 


\subsection{Les interruptions dans Sarkolène - esquisse}

Nous nous contenterons ici d'indiquer brièvement ce que pourrait être une étude interactionnelle des interruptions dans ce débat. Commençons par indiquer quelques-unes de ses propriétés les plus marquantes.

Comme tous les débats d'entre-deux-tours des présidentielles en France, celui-ci est structuré par des macro-tâches correspondant à autant de sujets à aborder, avec un ordre défini, et dans le respect d'un équilibre des temps de parole alloués aux deux candidats. Malgré ce contrôle, le débat de 2007 comporte beaucoup plus de turbulences que ceux de 1974, 1981, 1988 et 1995, se présentant comme une alternance de séquences monologales (durant lesquelles l'écouteur ne produit quasiment aucun régulateur verbal ni même non verbal, étant le plus souvent plongé dans la consultation de ses notes), et de passages dialogaux correspondant presque entièrement à des «zones interruptives», également pauvres en régulateurs verbaux et dans lesquels le taux d'interruptions coopératives est voisin de zéro (nos observations rencontrant à cet égard celles de Sandré 2009).

Dans l'ensemble du débat on peut distinguer en gros 18 grandes zones interruptives. La plus longue correspond, sans surprise, à la scène de la "saine colère " (7 minutes à peu près). Il n'y a aucune interruption dans les 15 premières minutes, aucune dans les 15 dernières, alors que les «zones » se succèdent sinon toutes les 5 minutes environ: sans doute parce qu'il est mal vu d'interrompre, et qu'il faut - pour faire preuve d'un bon ethos - produire en commençant une impression favorable, et la laisser en finissant.

Mais entre les deux, les infractions au système de l'alternance des tours se donnent plus libre cours, comme si elles devaient être un peu moins nuisibles pour l'ethos (mais on verra que celui de SR en a finalement pâti), ce malgré leur caractère majoritairement agressif parce que peu adouci : les " pré-interruptions » plus ou moins polies (vous permettez ? / si vous permettez... / pardon madame), les excuses, etc. sont tout à fait minoritaires dans le corpus. Néanmoins les passages de recouvrements plus faiblement interactifs n'en arrivent jamais au point du corpus $P h d e V$ : en tout état de cause, nous avons affaire à un corpus qui se maintient dans une sorte de position intermédiaire entre les deux extrêmes que nous avons envisagés plus haut.

\subsubsection{Types d'interruptions : une illustration}

Les interventions intercalaires, potentiellement interruptives, doivent être décrites en fonction :

- du degré de présence ou d'absence d'un TRP dans le tour interrompu ainsi que de sa nature (locale / globale) ;

- du degré de présence ou d'absence d'un TRP dans le tour interrupteur (vocation à conserver le tour ou non);

- du nombre de STI dans le tour interrompu / dans le tour interrupteur.

Illustrons cela à l'aide d'un extrait du débat Sarkolène, dont voici une transcription qui le segmente en STI (l'extrait commence à 01.56 .30 à la toute fin du 
long réquisitoire de SR contre l'immoralité de NS, et inaugure la scène de sa « saine colère ») :

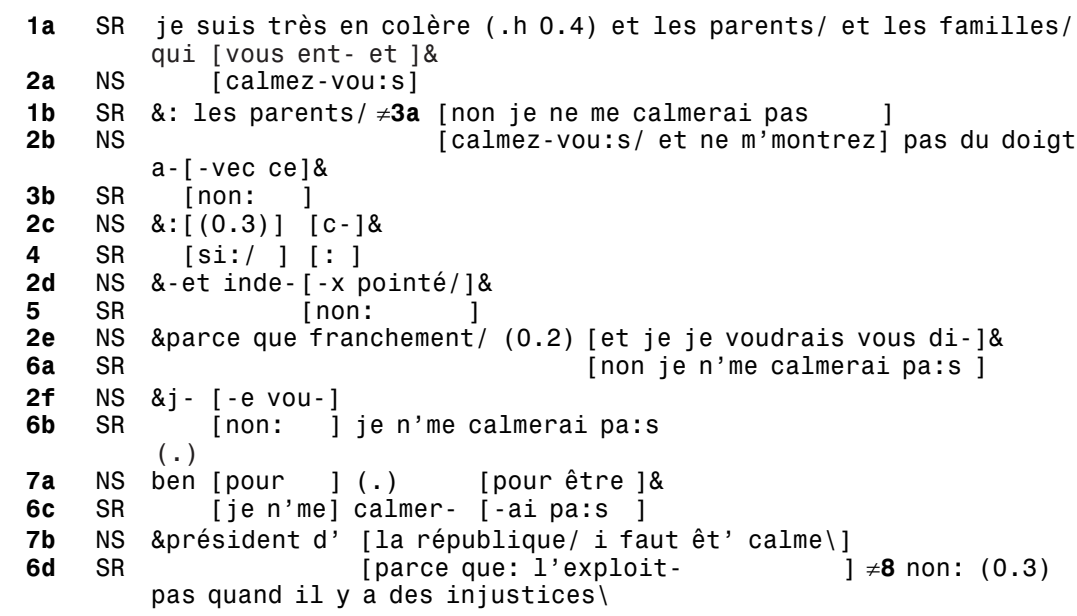

Les indices de STI attestent que tous les énoncés de l'extrait sont interrompus, à l'exception de deux interruptions brèves de SR (4 et 5). On a le plus grand nombre de STI dans le tour initialement interrupteur, celui de NS (2a-2f). NS montre plus de «suivi » que $\mathrm{SR}$, avec $2 \mathrm{TP}(2 \mathrm{a}-2 \mathrm{f}$ et $7 \mathrm{a}-7 \mathrm{~b})$ pour $8 \mathrm{STI}$, alors que pour SR on a 6 TP $(1 \mathrm{a}-1 \mathrm{~b}, 3 \mathrm{a}-3 \mathrm{~b}, 4,5,6 \mathrm{a}-6 \mathrm{~b}, 8)$ pour 11 STI. Elle se montre ainsi plus réactive à ce moment où sa grande offensive contre NS subit sa première contreattaque, et quant à NS il est plus offensif et plus insistant à ce point charnière du débat (et il peut se le permettre dans la mesure où il vient de subir sans broncher le long réquisitoire de sa rivale).

NS ne fait qu'une interruption à proprement parler (2a-2f), mais après l'avoir déployée par duplication ( $2 \mathrm{a}-2 \mathrm{~b})$, il parvient à la « tenir » envers et contre quatre interventions de SR, ce qui s'explique par le bénéfice d'une sorte de TRP global, en l'absence de TRP local (SR a bien assez parlé, elle a dit tout ce qu'elle avait à dire et commence à se répéter) : cas de unperturbated continuation s'il en est.

La première intervention potentiellement interruptive de SR (3a) intervient après un TRP de NS, auquel elle répond un peu à retardement, et elle ne se retrouve en chevauchement que parce que NS réitère son premier énoncé (2b); à ce détail près, elle intervient comme une réponse appropriée, un enchaînement plutôt qu'une interruption. Mais comme il s'agit d'un refus net, c'est-à-dire d'une répartie à caractère non coopératif, qui intervient à couvert d'un énoncé que NS donne comme suite à sa première intervention, et qu'en outre SR a déjà beaucoup parlé, elle paraît ne pas laisser continuer NS (les TRP globaux l'emportent sur les TRP locaux) et sa réplique, du moment que NS poursuit, peut produire un effet d'interruption illégitime (c'est ainsi que l'a perçue un transcripteur non spécialiste, voir ci-dessous). On peut a fortiori dire la même chose de ses autres interventions (les escarmouches centrales 4 et 5 , ainsi que $6 a-6 d$ qui est la réduplication de 3a-3b), sauf qu'elles aggravent son cas, faute d'intervenir à des TRP locaux, ce que NS rend patent en n'achevant pas son TCU de $2 \mathrm{f}$.

Nos distinctions sont ainsi utiles pour décrire des comportements interactifs saisis sur le moment. Mais elles le sont aussi pour saisir des tendances au long 
cours et mettre en évidence des comportements spécifiques, à verser au compte de l'ethos des participants. Remarquons à ce titre que si les deux débatteurs s'interrompent à qui mieux mieux, ce n'est pas forcément ce que l'on retient. En témoigne une transcription de ce passage trouvée sur un forum de discussion ${ }^{(5)}$ :

- Et je trouve que la façon dont vous venez, de nous décrire, comme ça, *tape sur la table* la larme à l'œil, les droits des enfants d'handicapés à intégrer les écoles [...] est scandaleux. [...]

- Calmez-vous, calmez-vous.

- (en même temps, coupant la parole) NON je ne me calmerai pas.

L'intervention de Sarkozy («Calmez-vous, calmez-vous ») n’est pas retenue comme interruptive, alors qu'elle fusionne deux énoncés produits, dans les faits, en chevauchement - comme si c'était Royal qui interrompait Sarkozy et non l'inverse...

\subsubsection{Profils interruptifs de SR et NS}

Il découle de ce qui précède qu'il y a trois possibilités pour décrire les rapports entre le(s) tour(s) de A et le(s) tour(s) de B dans les zones interruptives :

- le recouvrement : A et B poursuivent parallèlement leurs tours respectifs sans prendre en compte celui de l'autre ; les tours comportent souvent plusieurs TCU, éventuellement à rallonge, mais ne sont pas décomposables en STI, ce qui apparaît dans les transcriptions par des «" ", "” " etc. ;

- le tuilage : A et B s'interrompent alternativement tout en cédant rapidement le tour, en fin de TCU ou non ; il y a des interruptions alternées mais sans STI ;

- le saupoudrage: A poursuit son tour tandis que B multiplie les interruptions brèves, soit composées d'un TCU achevé et comportant alors un TRP, soit s'arrêtant en cours de TCU; il y a de nombreux STI chez A, ce qui produit un effet de parole usurpée.

C'est tendanciellement la troisième de ces possibilités qui caractérise le plus NS en tant qu'interrupteur, tandis que SR est plutôt spécialisée dans la première. Voici un extrait caractéristique (Sarkolène 01.51.00) :

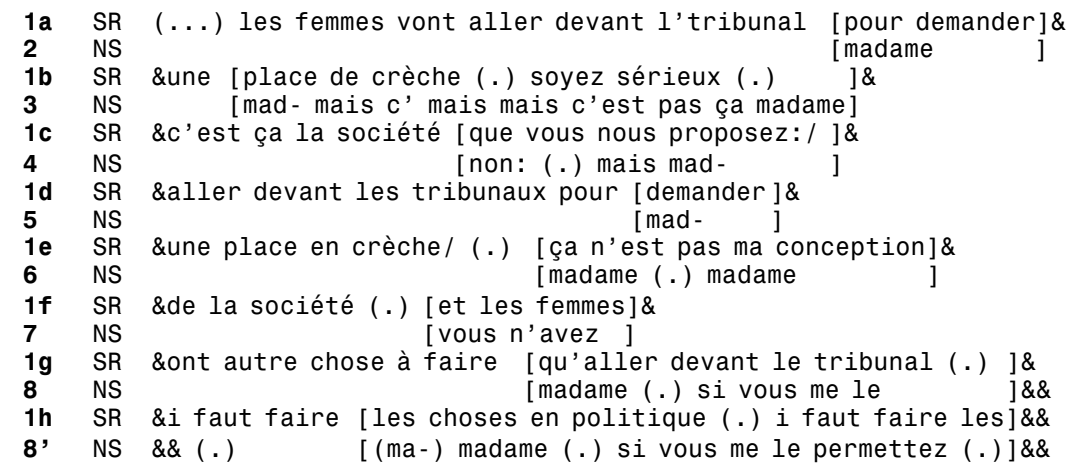

(5) http://www.cyberados.com/lofiversion/index.php/t7068.html 
1h' SR \&[choses (.) c'est pas le tribunal ]

8', NS \&\&[vous n'avez pas besoin ] d'être méprisante pour être brillante (.) voilà

On a là un cas de figure typique de ce débat. Les six premières interruptions de NS $(2,3,4,5,6,7)$, placées en dehors de tout TRP détectable chez SR, sont brèves et ne font qu'ébaucher des TCU qui restent inachevés; du moment que SR continue imperturbablement (pas une seule prise en compte de NS dans le long tour 1a-1h', pas une seule réparation, pas une seule variation de débit ni d'intensité), on a l'impression qu'elle ne le laisse pas parler, un peu comme si c'était elle qui, par six fois, l'interrompait. Ce saupoudrage initial de la part de NS prépare le terrain pour sa septième interruption $(8-8$,$) qui, après une phase de recouvrement,$ s'impose. Le rapport tours / STI est éloquent : avec 8 tours et 8 segments (mais à proprement parler aucun «vrai » STI, puisque ce sont des tours mono-segment) contre 1 tour et 8 STI, NS est celui qui interrompt le plus ${ }^{(6)}$ mais qui paraît le plus interactif : ceci compensant largement cela du double point de vue de la légitimité de la parole et de l'ethos.

Les commentaires a posteriori à propos de ce débat reflètent cette ambivalence des interruptions de NS et de la résistance de SR (sa "pugnacité », dirait $\mathrm{OM}$ ), ainsi dans cette remarque lue sur un autre forum ${ }^{(7)}$ :

Elle reprochait à Sarko de lui couper la parole alors que c'est elle qui l'interrompait tout le temps et impossible de l'arrêter !!!

Ce que ce commentaire pointe, c'est une stratégie consistant à se présenter comme la victime des interruptions d'autrui, ce que Luginbühl (2007 :1380) appelle doing being interrupted, en précisant que les protestations de type «please let me finish my statement, I let you finish », quasiment ritualisées dans les débats, ne recouvrent souvent qu'une accusation non justifiée, et ne sont qu'une technique pour discréditer l'adversaire et son comportement :

this method has become frequent practice in cases where there is not first interruption, but rather short objections, simultaneous starts or similar interventions that only resemble interruptions but do not claim the turn. Indeed, even persons who only make one short objection are sometimes addressed as permanent interruptors.

Mais on peut aussi remarquer une petite contradiction dans le double reproche « elle l'interrompait tout le temps et impossible de l'arrêter » : impossible de l'arrêter ? C'est reconnaître qu'on - et surtout NS - a dû essayer à maintes reprises, sans parvenir à lui « couper la chique ». À l'examen, ces tentatives s'avèrent au-

(6) C'est d'ailleurs ce qui ressort de nos propres décomptes sur l'ensemble du débat : plus de 220 interruptions pour NS, environ 140 pour SR - mais les décomptes sont très sujets à caution (M. Sandré, rappelons-le, obtient des chiffres sensiblement différents), sans que cela invalide pour autant la notion d'interruption : comme il s'agit d'un phénomène graduel, on ne peut pas s'étonner de trouver des variations dans son appréciation. Aussi, ce qui importe bien davantage que les décomptes, c'est de décrire les types d'interruption majoritairement rencontrés.

(7) http://www.lepoint.fr/actualites-politique/2007-05-03/le-duel-royal-sarkozy-decryptepar-joseph-messinger-specialiste/917/0/181429 
tant d'interruptions, c'est-à-dire de prises de parole de NS pendant un TCU en cours de SR sans qu'on puisse déceler aucun TRP (du moins « local»). Mais l'effet produit, c'est que SR à ces moments " ne le laisse pas en placer une » : la parole ininterrompue produit en somme les mêmes effets que l'interruption.

\section{Conclusion}

Le tour [...] est accompli par les participants qui déploient séquentiellement une série d'indications rendant manifeste si le tour est possiblement complet [...] ou s'il se poursuit [...]. C'est ainsi que la forme qui est conférée au tour exhibe ses points de complétude ou d'incomplétude [...]. Les interruptions sont elles-mêmes des indices reconnaissables de la façon dont les participants suivent le déroulement du tour (Gülich et Mondada $2001: 207$ et 209 ; italique ajouté)

Outre que les interruptions peuvent être au contraire dans certains cas des indices de la façon dont les participants ne suivent pas le déroulement du tour ou tout au moins, n'en tiennent aucun compte, de telles affirmations peuvent sembler exagérément optimistes : si certains indices et marqueurs, sur lesquels il est du devoir de l'analyste de s'appuyer, sont en effet inscrits dans le texte de l'interaction, ils ne sont jamais « transparents »; ce sont des signifiants qui s'offrent à l'analyste (comme aux participants eux-mêmes), et la tâche consistant à leur assigner certaines valeurs particulières ne va jamais de soi. Cela vaut tout particulièrement pour les types d'unités dont il est ici question : les TP, TRP, TCU et autres STI sont, on l'a vu, tout sauf « exhibés ». On retrouve ici le principe de gradualité : les marqueurs sont plus ou moins évidents, et même les plus manifestes en apparence (comme les énoncés métacommunicatifs tels que «attendez », " je n'ai pas terminé », ou « pardon de vous interrompre ») doivent eux aussi être passés au crible de l'analyse ${ }^{(8)}$.

Le travail interprétatif - pour les participants « actifs » et leur auditoire, comme pour cet « archi-interprétant » qu'est l'analyste - concerne d'abord, on l'a vu, l'identification elle-même des interruptions (en relation avec celle des tours et des TRP) ; mais il concerne aussi leurs valeurs et effets interactionnels, lesquels engagent un certain nombre de distinctions (toutes également graduelles) que nous ne pouvons ici que rappeler rapidement :

- interruptions « coopératives »vs « polémiques » (Sandré 2009), « supportives »vs « compétitives » (Bazzanella 1991), « affiliatives »vs « disaffiliatives » (Goldberg 1990);

- interruptions perçues comme « offensives » ou au contraire « défensives » (Agha $1997: 475)$;

- interruptions plus ou moins « polies », entre autres par la façon dont elles sont préfacées ou "réparées » (voir le recours systématique, par Sarkozy face à Royal, à des formules telles que « madame si vous me permettez de terminer » ou le fameux « est-ce que vous souffrez que je puisse faire une phrase ») ;

(8) Ce que nous avons fait pour le corpus « Sarkolène », sans qu'il soit ici possible ici de présenter cette analyse. 
- interruptions plus ou moins « légitimées 》 (par le caractère « intarissable » de A, le fait qu'il/elle transgresse ostensiblement telle ou telle maxime conversationnelle, ou qu'il/elle attaque B de façon excessivement violente, insultante ou diffamatoire...)

Tous ces aspects doivent être envisagés en relation avec le type d'interaction auquel on a affaire - dans les débats, qui sont des sortes de " guerres verbales », les interruptions vont dans leur grande majorité être utilisées comme des armes stratégiques alors que dans d'autres contextes, comme les conversations familières, elles sont plutôt à mettre à mettre en corrélation avec le degré d' " engagement » dans l'échange et de chaleur dans la relation (Tannen 1989) ${ }^{(9)}-$, mais aussi avec les caractéristiques propres de l'interaction en cours et le turn-taking style (Beattie 1982) des parties en présence.

En tout état de cause, quel que soit l'angle sous lequel on choisit de s'intéresser aux interruptions, leur étude a tout intérêt à recourir aux outils descriptifs élaborés dans le cadre de l'analyse conversationnelle, dont elles permettent précisément de mettre en évidence le caractère «élaboré » (rien de moins «naturel» que la notion de tour de parole...). Introduisant un certain " désordre » dans le fonctionnement de la machinerie conversationnelle, ces empêcheurs de tourner en rond que sont les phénomènes interruptifs constituent à la fois, pour les participants à l'interaction, une ressource leur permettant de s'émanciper dans une certaine mesure, et à des fins variables, du carcan du turn-system, et pour les analystes de l'interaction, une source inépuisable de questionnements.

(9) Rappelons que la fréquence comme la valeur des interruptions dans les conversations familières sont soumises à d'importantes variations culturelles, ainsi que l'ont montré un certain nombre d'études. 


\section{Bibliographie}

AghA, A. (1997) : « Tropic aggression in the Clinton-Dole presidential debate », Pragmatics 7, 461-497.

BAZZANELla, C. (1991) : «Le interruzioni "competitive" et "supportive". Verso una configurazione complèssa », in $\mathrm{S}$. Stati, E. Weigand et F. Hundsnurscher (éds), Dialoganalyse III, Teil 1, Tübingen, Niemeyer, 283-305.

BEATTIE, G.W. 1982 : « Turn-taking and interruption in political interviews : Margaret Thatcher and Jim Callaghan compared and contrasted », Semiotica 39 (1/2), 93-113.

CONSTANTIN DE CHANAY, H. et KeRBRAT-ORECCHIONI, C. (2007) : « 100 minutes pour convaincre : l'éthos en action de Nicolas Sarkozy », in M. Broth et al. (éds), Le français parlé des médias, 309-329, Stockholm, Acta Universitatis Stokholmiensis, 309-329.

FORD C.E., FOX, B.A. et THOMPSON, S.A. (1996) : «Practices in the construction of turns : the 'TCU' revisited », Pragmatics 6 (3), 427-454.

GOLDBERG, J.A. (1990) : «Interrupting the discourse on interruptions », Journal of Pragmatics 14 (6), 883-903.

Goodwin, M.H. et Goodwin, C. (1986) : « Gesture and Coparticipation in the Activity of Searching for a Word », Semiotica 62, 51-75.

GREVISSE, M. (1986) : Le bon usage. Douzième édition refondue par André Goose, Louvain-la-Neuve, Duculot.

GuiLlot, M.-N. (2005) : « Revisiting the methodological debate on interruptions : From measurement to classification in the annotation of data for cross-cultural research », Pragmatics 15 (1) : 25-48.

GÜlich, E. et MondadA, L. (2001) : «Konversationsanalyse », in G. Holtus, M. Metzeltin et Schmitt, C. (éds), Lexicon der Romanistischen Linguistik, vol. I (2), Tübingen : Niemeyer, 196-252.

HUTChBy, I. (1992) : "Aspects of "interruption" in argument sequences on talk radio », Text 12 (3), 343-471.

JEFFERSON, G. (1972) : «Side sequences », in D. Sudnow (éd.), Studies in social interaction, New York, Free Press, 294-338.

LERNER, G.H. (1989) : «Notes on Overlap Management in Conversation : The Case of Delayed Completion », Western Journal of Speech Communication 53, 167-177.

LUGINBÜHL, M. (2007) : «Conversational violence in political TV debates : Forms and functions », Journal of Pragmatics 39, 1371-1387.

MONDADA, L. (2001) : «Pour une linguistique interactionnelle», Marges linguistiques 1, mai 2001, 142-162. (http://www.marges-linguistiques.com)

- (2004) : «Temporalité, séquentialité et multimodalité au fondement de l'organisation de l'interaction : Le pointage comme pratique de prise du tour », CLF 26, 269-292.

— (2007) : «L'organisation online par les co-participants de la structuration du tour in fieri en TCUs : évidences multimodales », TRANEL 47, 7-38.

MurRay, S.O. et COVELli, L.H. (1988) : « Women and men speaking at the same time », Journal of Pragmatics 12 (1), 103-111.

SACKS, H. (1992) : Lectures on Conversation, vol. I, Oxford, Basil Blackwell. 
SACKs, H., Schegloff, E. A. et Jefferson, G. (1974) : «A Simplest Systematics for the Organization of Turn-Taking in Conversation », Language 50, 696735.

SANDRÉ, M. (2009) : "Analyse d'un dysfonctionnement interactionnel - l'interruption - dans le débat de l'entre-deux-tours de l'élection présidentielle de $2007 »$, Mots 89, 69-82.

SCHEGLOFF, E.A. (1987) : «Recycled turn beginnings : A precise repair mechanism in conversation's turn-taking organization », in G. Button et J.R. Lee (éds), Talk and Social Organisation, Clevedon, Multiligual Matters, 70-85.

— (1992) : " To Searle on Conversation: A Note in Return », in J.R. Searle, H. Parret et J. Verschueren, (On) Searle on Conversation, Amsterdam/Philadelphia : John Benjamins, 113-128.

- (2001) : «Accounts of Conduct in Interaction : Interruption, Overlap, and Turn-Taking », in J.H. Turner (éd.), Handbook of Sociological Theory, New York : Plenum, 287-321.

SCHMALE, G. (2002) : «Allô, tu m'entends ? Intercompréhension en communication téléphonique », Actes de la journée d'étude "Autour du dialogue », Lyon, 6 décembre 2002, LYLIA 4,

http://langues.univ-lyon2.fr/1134-Journees-d-etude-Autour-dialogue.html

- (2008) : "Constructions inachevées et transfert du tour de parole », in J. Durand, B. Habert et B. Laks, CMLF 2008, Paris, ILF, 817-834.

SElting, M. (2000) : "The construction of "units" in conversational talk ", Language in Society 29, 477-517.

TALBOT, M. (1992) : «I wish you'd stop interrupting me ! Interruptions and asymmetries in speaker-rights in equal encounters », Journal of Pragmatics 18, 415-466.

TANNEN, D. (1989) : «Interpreting Interruption in Conversation », in Papers from the 25th Annual régional Meeting of the Chicago Linguistic Society, Part II : Parasession on Language in Context, Chicago, Chicago Linguistic Society, 266-287.

Trognon, A. (1988) : " Passages à l'acte dans les entretiens d'enquête », Connexions $52: 67-80$.

Trognon, A. et Larrue, J. (1994) : Pragmatique du discours politique, Paris, A. Colin.

West, C., Zimmerman, D.H. (1985) : « Gender, Language and Discourse », in T.A. van Dijk (ed.), Handbook of Discourse Analysis, vol. 4, 103-124.

ZIMMERMAN, D.H. et WEST, C. (1975) : « Sex roles, interruptions and silences in conversation ", in B. Thorne et N. Henley (éds), Language and Sex : Difference and Dominance, Rowley, MA, Newbury House, 105-129. 


\section{Conventions de transcription}

Nous utilisons les conventions de transcription ICOR du laboratoire ICAR dirigé par Lorenza Mondada. Voici les principales :

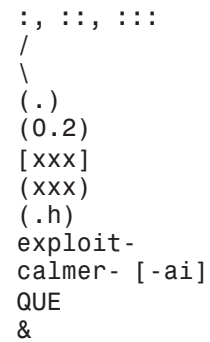

Pour plus de précisions sur les conventions de transcription ICOR, voir http://icar.univ-lyon2.fr/projets/corinte/bandeau_droit/convention_icor.htm/ Nous commentons l'usage particulier que nous faisons du signe de différence $\ll \neq »$ en 2.2.1. 